\title{
Intracranial MR Venography Using Low-Field Magnet: Normal Anatomy and Variations in Nepalese Population
}

\author{
Sharma UK' , Sharma K² \\ 'Department of Radiodiagnosis, ${ }^{2}$ Department of Neurosurgery, B \& B Hospital, Gwarku, Lalitpur, Nepal
}

Introduction: Magnetic resonance (MR) venography is considered a reliable imaging modality for the evaluation of intracranial venous system. The purpose of this study was to evaluate the normal venous anatomy and its variations in Nepalese population using low field MR technique.

Methods: One hundred patients with normal MR imaging of brain underwent MR venographic study. MR venograms were performed in $0.35 \mathrm{~T}$ MR scanner using a contiguous 2D time-of-flight MR angiographic technique.

Results: The flow gaps in the transverse sinus were seen in $47 \%$ of population, of which $91 \%$ occurred in the non-dominant side. Right transverse sinus was dominant in $73 \%$ population. Flow gap was observed in bilateral transverse sinus in one case, while it was seen in the dominant right transverse sinus in $6.3 \%$ population. Inferior sagittal sinus was observed in $11 \%$ cases. Internal cerebral vein was seen in 60 cases. Occipital sinus was observed in $4 \%$ of the cases. The basal vein of Rosenthal was observed in $34 \%$ of the cases, whereas vein of Labbe seen in $8 \%$ cases. Unilateralflow gap in the sigmoid sinus was seen in 5 cases.

Conclusions: MR angiography done at low field MR unit is a reliable tool in cerebral venous sinus assessment, particularly major dural sinuses. The flow gaps in transverse sinus are frequently encountered anatomic variation. Visualization of small veins like inferior sagittal sinus, basal vein of Rosenthal, vein of Labbe, internal cerebral vein was inferior in our study compared to other studies done in high field MR unit.

Keywords: Dural sinus, intracranial veins, MR venography

\section{INTRODUCTION}

Intracranial sinus and venous anatomy can be visualized by several imaging techniques, including conventional angiography, CT venography and MR venography (MRV). MRV is a widely available, reproducible, noninvasive means of evaluating venous anatomy. The disadvantages of the conventional and CT angiography are the use of ionizing radiation and the invasiveness of procedures with its inherent applications. Therefore, MRV has got several advantages over other imaging modalities. In MRV the image of venous system can be acquired with or without the use of contrast media.

\footnotetext{
Correspondence:

Dr. Umesh Kumar Sharma

B \& B Hospital

Gwarku, Lalitpur

Nepal

Tel no: 977-1-9851071500

E-mail:dr_uksharma@yahoo.com
} 


\section{METHODS}

Patients who were sent for MRI examination of brain in the Department of imaging, B \& B hospital from January 2011 to August 2011 were enrolled in this study. Patients with any congenital or acquired intracranial abnormality, venous thrombosis or previous surgery were excluded from the study. First one hundred patients who had no abnormal findings on MR examinations of brain were included in the study and subjected to MR venography. Cerebral MR venography was performed after acquisitions of routine sequences. MR venograms were performed in 0.35 Tesla MR scanner (Siemens magnetom C) using a contiguous 2D time-of-flight (TOF) MR angiographic technique. Sections with thickness of $3.5 \mathrm{~mm}$ were acquired in the coronal plane using the following parameters 50/9.24 (TR/TE), 60 degree flip angle, $230 \mathrm{~mm}$ field of view, and 156x256 matrix. All MR venographic source images, thus obtained, were post-processed using the maximum pixel intensity projection (MIP) method, generating 19 MIP projections at 10 degree increments. Resulting venograms were reviewed in each case for the presence or absence of the image-intracranial veins, including the internal cerebral veins, the basal veins of Rosenthal, and the vein of Galen.

\section{RESULTS}

Magnetic resonance venograms could be obtained from all 100 patients with normal MR examinations of brain parenchyma. Superior sagittal sinus (SSS) was seen in every single case (Figure 1). Short segment flow gap was found in SSS in 5 cases.

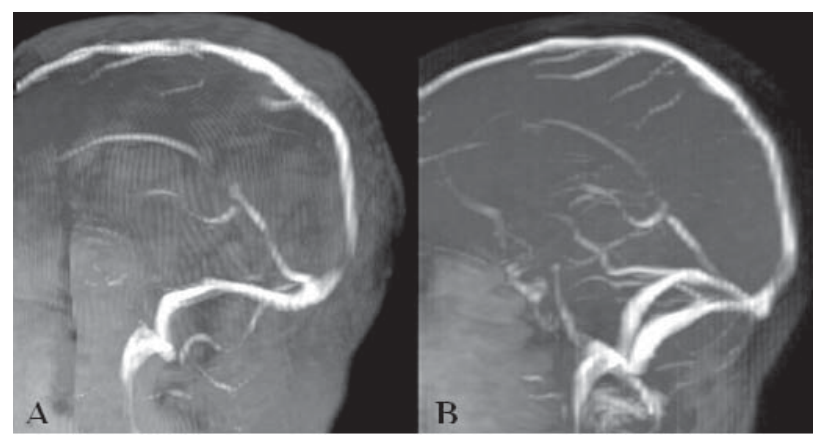

Figure 1. MRV showing Superior sagittal sinus, inferior sagittal sinus, straight sinus, vein of Galen, internal cerebral vein

Straight sinus was completely visualized in $91 \%$ cases while incompletely visualized in 9 cases. Details of the visualized veins are given in table 1. Vein of Galen was not seen in 4 cases. Right transverse sinus was dominant in $73 \%$, left sided transverse sinus was dominant in $18 \%$ and co-dominant sinuses were seen in $9 \%$. Flow gaps within the transverse sinuses were observed in $47 \%$ of the subjects (Figure $2,3,4$ ).

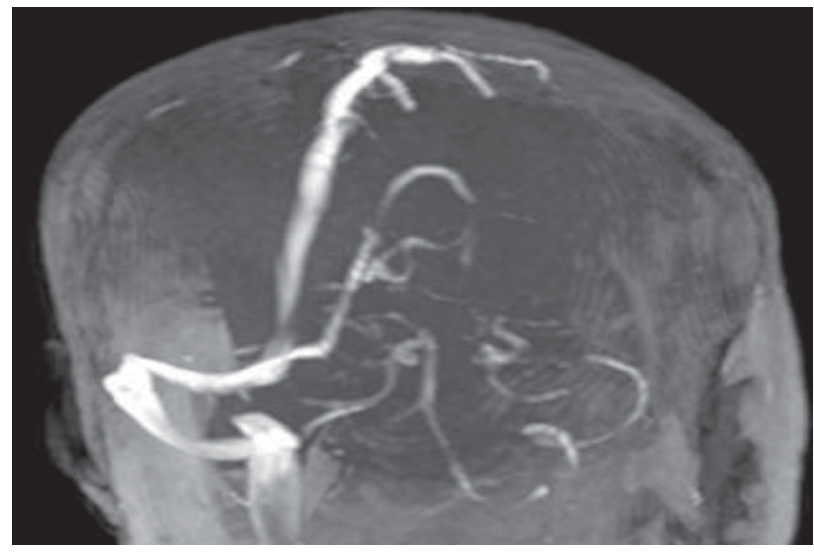

Figure 2. Hypoplastic left transverse sinus and nonvisualised internal jugular vein

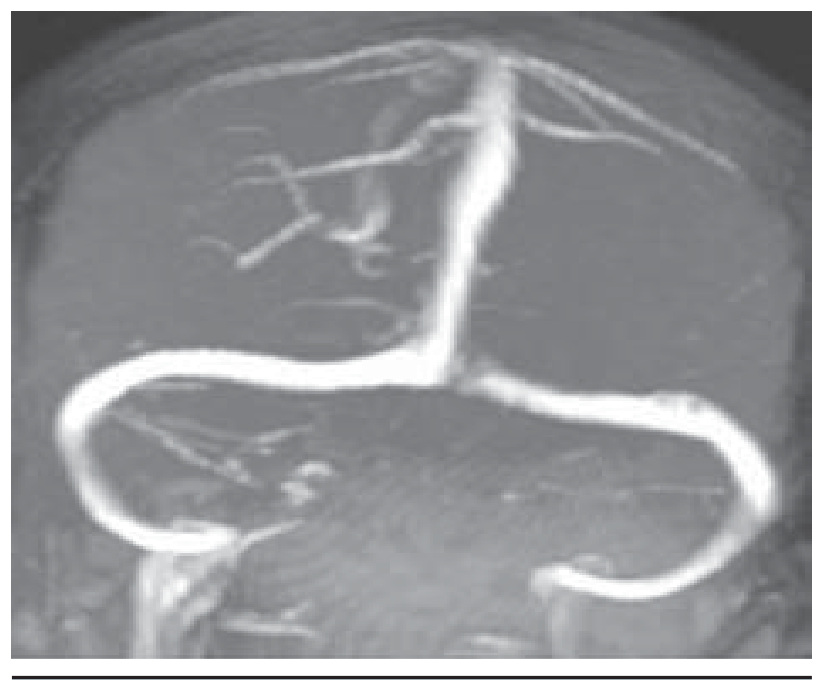

Figure 3. Flow gap in left transverse sinus

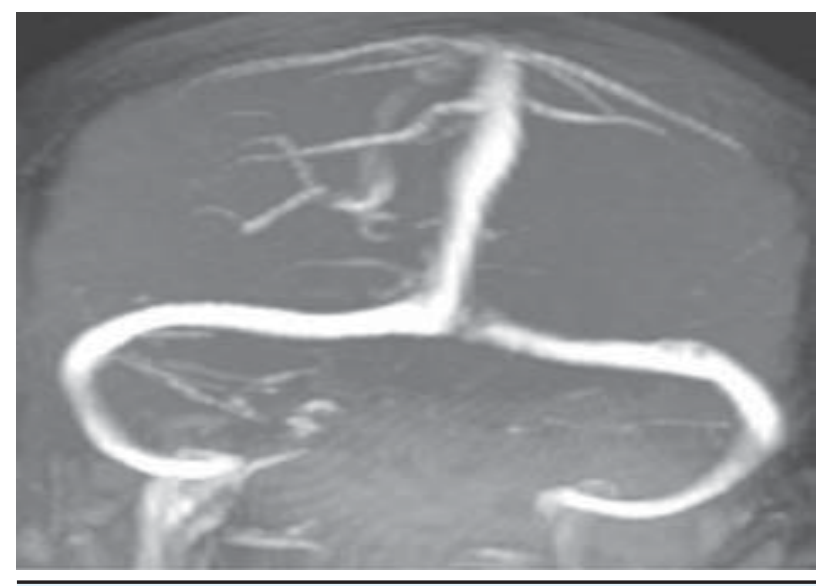

Figure 4. Codominant transverse sinuses

Of these, $91.4 \%(43 / 47)$ occurred in the non-dominant left transverse sinus, $6.3 \%(3 / 47)$ cases showed flow 
gap on dominant right transverse sinus, whereas flow gap was observed in bilateral transverse sinus in one case. In 43 cases with flow gap in left transverse sinus, the flow gap in 13 cases has more than $1 / 3^{\text {rd }}$ of the length and less than $1 / 3^{\text {rd }}$ length in 30 cases (Figure 5 ).

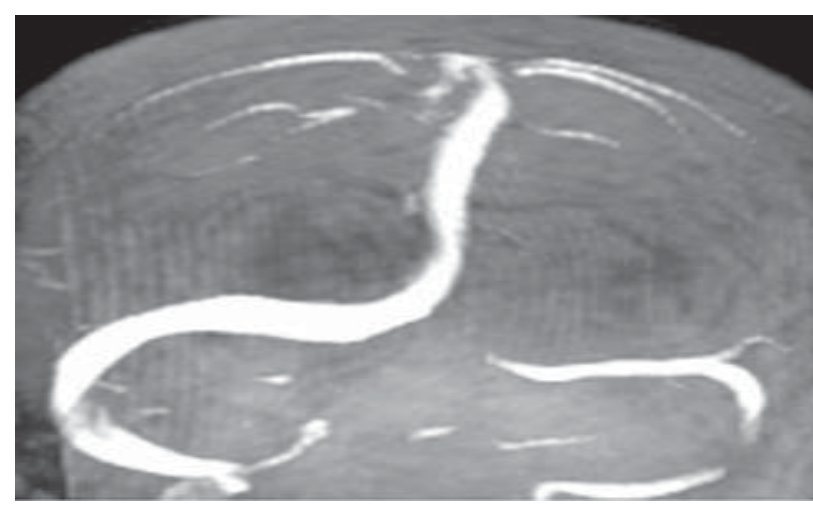

Figure 5. Flow gap in non-dominant left transverse sinus

Inferior sagittal sinus (ISS) was observed in $11 \%$ cases. Internal cerebral vein(ICV) was seen in 60 cases. An occipital sinus was observed in $4 \%$ of the cases (Figure $6)$.

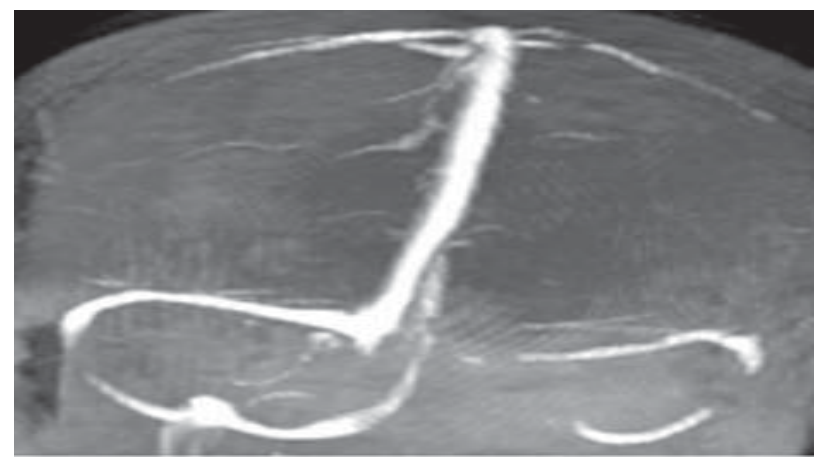

Figure 6. MRV showing occipital sinus

Table 1. Visualization of the intracranial veins

\begin{tabular}{lcc}
\hline Paired structures & Right & Left \\
\hline Transverse sinus & 100 & 100 \\
\hline Sigmoid sinus & 97 & 98 \\
\hline Internal jugular vein & 90 & 93 \\
\hline Internal cerebral vein & 60 & 60 \\
\hline Vein of Rosenthal & 34 & 34 \\
Vein of Labbe & 8 & 8 \\
\hline Vein of Galen & 96 & 96 \\
\hline Unpaired structures: & & \\
\hline Superior sagittal sinus & & 100 \\
\hline Inferior sagittal sinus & & 11 \\
\hline Straight sinus & & 91 \\
\hline
\end{tabular}

The basal vein of Rosenthal was observed in $34 \%$ of the cases, whereas vein of Labbe wasseen in $8 \%$ cases only. Unilateral flow gap in the sigmoid sinus was seen in 5 cases.

\section{DISCUSSION}

The aim of this study was to evaluate the normal anatomic variations in intracranial venous system in the Nepalese population. The venous system has been imaged with conventional angiography, but MR venography has increasingly been used to study the cerebral venous system. MR venography is noninvasive, does not involve ionizing radiation, and can be performed at the same time as MR imaging in comparatively short acquisition times. ${ }^{1}$

The commonly applied MR methods of evaluating dural venous sinuses are $2 \mathrm{D}$ or $3 \mathrm{D}$ phase contrast enhanced thin sections gradient echo, and 2D MRV techniques. ${ }^{2,3,4}$ Phase contrast venography is limited by a potential lack of sensitivity to flow because of the selection of an inappropriate velocity encoding value and its relatively lengthy acquisition times. ${ }^{4,5}$ Compared with 2D TOF MRV, 3D contrast enhanced gradient echo techniques are more sensitive to dural sinus thrombosis, they are less affected by saturation effects, and they enable better differentiation of atretic and hypoplastic sinuses. ${ }^{3}$

Major cerebral venous sinuses are often studied by using the two dimensional time-of flight (2D-TOF) MRV. ${ }^{6,7}$ Liauw et al claimed, that three dimensional phasecontrast MR angiography (3D PC-MRA) and 2D-TOF MRA techniques are equally good for visualization of the intracranial venous sinuses. PC MRA is very sensitive in cases of subacutedural sinus thrombosis, where T1 W hypersensitivity is falsely suggested as patent sinus, in the 2D TOF technique. ${ }^{7}$

Contrary to other studies which were done on 1.5T MR units using the 2D TOF technique, our study is based on acquisition of images in low field permanent magnet $0.35 \mathrm{MR}$ units

Although 2D TOF imaging is exquisitely sensitive to slow flow, it nevertheless has a minimum threshold below which sufficient signal from flowing blood cannot be obtained. To overcome artifactual signal loss resulting in signal loss from in plane vascular flow, it is desirable to orient the acquisition plane perpendicular to the long axis of the vessel being imaged. Because the bulk of the intracranial venous flow is in an anteroposterior direction, toward the torcular Herophili, since acquisitions in the coronal plane satisfy the requirement of perpendicular orientation during acquisition for a majority of the intracranial dural sinuses. Complex flow patterns, such as flow separation with vortex flow, can 
generate hemodynamic conditions that are difficult to image and that may contribute to intravascular signal loss. ${ }^{8}$

Time-of-flight technique rely mainly on flow-related enhancement for producing vascular images, phasecontrast MR angiography uses velocity-induced phase shifts imparted upon the moving spins to distinguish flowing blood from the surrounding stationary tissue. Although excellent background suppression is a major advantage, the quantitative determination of blood velocities may be possible, phase-contrast MR angiography may require long imaging times and priori estimate of blood flow velocity. It may also be more sensitive to signal loss due to turbulence or intravoxel dephasing. $^{8}$

In the study reported by Ayanjen et al, thirty-one per cent of their patient population had flow gaps in the transverse sinus, $90 \%$ of which were in the nondominant sinus which are potentially indistinguishable from the thrombosis ${ }^{8}$. Alper et al, described flow gaps in $24 \%$ of normal population. ${ }^{9}$ Our study revealed flow gaps in the transverse sinus in $47 \%$ of population, of which $91 \%$ occurred in the non-dominant side. In the study right transverse sinus was dominant in $73 \%$ population. Flow gap was observed in $\mathrm{B} / \mathrm{L}$ transverse sinus in one case, where as in the dominant right transverse sinus in $6.3 \%$ population.

Alper et al described the transverse sinus abnormalities i.e. $20 \%$ aplasia and $39 \%$ hypoplasia of the left sinus, $6 \%$ hypoplasia and $4 \%$ aplasia of the right sinus and $31 \%$ symmetric sinus. ${ }^{9}$ Our results also showed most of the hypoplastic and aplastic transverse sinuses on the left side only. During fetal development as the volume of the telencephalon increases, the torcula descends resulting in the inferior inclination of the lateral portion of the transverse sinuses which becomes less marked. During the period of transition from the stage of plexiform networks to that of regular transverse sinus there is rapid increase and then decreased in caliber occurs in the region of the torcula and the lateral sinus, may in some cases become irregular or the lateral sinus may be markedly hypoplastic or may even be absent in its major portion, less commonly, the lateral portion of the transverse or sigmoid sinus may also be hypoplastic or absent. ${ }^{9}$

In a study by Widjaja et al, occipital sinuses were reported in $18 \%$. Our study showed occipital sinus in only $4 \%$ of cases $^{1}$. In $10 \%$ of the cases, occipital sinuses were shown in MR venograms obtained from 100 patients ranging in the age from 9 days to 83 years. ${ }^{8}$ Other authors have reported the presence of occipital sinuses in $35.5 \%$ of cases. ${ }^{1,10}$

In the study by Ayanjen et al superior sagittal sinus and straight sinus, vein of Galen were observed in the all the cases. ${ }^{8}$ Inferior sagittal sinus was seen in $52 \%$ of the studies, where as internal cerebral vein, vein of Rosenthal in $91 \%$ of cases. But our study showed short segment flow gap in superior sagittal sinus in 5 cases. ISS was seen in $11 \%$, ICV in $60 \%$, vein of Rosenthal in $34 \%$ of cases. The difference in observation in our study could be related to the low field MR unit and related signal loss or artifacts.

Limited number of the cases and study being carried out from single center is the limitation of this study. Study from more centers are required to see more variations if there is any.

\section{CONCLUSION}

It is important to recognize the normal variation of cerebral dural sinuses and not mistake for pathologic abnormalities. Flow gap in the venous sinus, hypoplasia and aplasia of the transverse sinus have been reported in the various literatures and should not be mistaken for thrombosis. The study showed MR angiography done at low field MR unit a reliable tool in cerebral venous sinus assessment, particularly for major dural sinuses. Flow gap in the transverse sinus was commonly encountered anatomic variations. However visualization of small veins like inferior sagittal sinus, basal vein of Rosenthal, vein of Labbe, internal cerebral vein was inferior in our study compared to that of other studies done in high field MR unit. 


\section{REFERENCE}

1. E. Widjaja, P.D.Grifiths. Intracranial MR venography in children: Normal anatomy and variations. Am J Neuroradiol 2004;25:1557-62

2. Rollins $\mathrm{N}$, Ison $\mathrm{C}$, Booth $\mathrm{T}$, Chia J. MR venography in the pediatric patient. Am J Neuroradiol 2005;26:50-5

3. Liang L, Korogi $\mathrm{Y}$, Sugahara $\mathrm{T}$, Ikushima I, Shigematsu Y, Takahashi M et al. Normal structures in the intracranial dural sinuses: delineation with 3D contrast-enhanced magnetization prepared rapid acquisition gradient-echo imaging sequence. Am J Neuroradiol 2002;23:1739-46

4. Rippe DJ, BoykoOB, Spritzer CE,Meisler WJ, Dumoulin CL, Souza SPet al. Demonstration of dural sinus occlusion by the use of MR angiography. Am J Neuroradiol 1990;11:199-201

5. Nadel L, Braun IF, Kraft KA, Fatouros PP, Laine FJ. Intracranial vascular abnormalities: value of MR phase imaging to distinguish thrombus from flowing blood. AJR 1991;156:373-80
6. Mattle HP, Wentz KU, Edelman RR, Wallner B, Finn JP, Barnes $\mathrm{P}$ et al. Cerebral venography with MR. Radiology 1991;178:453-8

7. Liauw L, van Buchem MA, Split A, de Bruine FT, van den Berg R, Hermans J et al. MR angiography of the intracranial venous system. Radiology 2000;214:67882

8. Ayanzen RH, Bird CR, Keller PJ, Mc Cully FJ, Theobald MR, HeisermanJEet al. Cerebral MR venography: Normal anatomy and potential diagnostic pitfalls. Am J Neuroradiol 2000;21:74-8

9. Alper F, Kantarci M, Dane S, Gumustekin K, Onbas $\mathrm{O}$, Durur I. Importance of an anatomical asymmetries of transverse sinuses: An MR venographic study. Cerebrovasc Dis 2004;18:236-9

10. Lang J. The floor of the posterior cranial fossa. In: clinical Anatomy of the posterior cranial fossa and its foramina. New York: Thieme; 199:6-9 